\title{
EUROPEAN HEAVY ION ICF DRIVER DEVELOPMENT
}

\author{
G. PLASS, CERN, Geneva, Switzerland
}

\begin{abstract}
Approaches in Europe to heavy ion induced Inertial Confinement Fusion are oriented toward the linac-plusstorage ring technique. Despite the very limited support of this work, technical progress was achieved in some important areas. For the immediate future, a substantial intensity upgrade of the GSI accelerator facilities at Darmstadt is being implemented, leading to specific energy depositions of the order of $100 \mathrm{~kJ} / \mathrm{g}$ and plasma temperatures of 10 to $20 \mathrm{eV}$. For the longer term, a conceptual design study of a heavy ion based Ignition Facility is being initiated.
\end{abstract}

\section{INTRODUCTION}

The quest for 'new' or 'alternative' energy sources for mankind is getting a new impulse since the late eighties through the increasing concern about the climatic consequences of the ever growing use of fossile fuels and the consequential increase of $\mathrm{CO} 2$ concentration in our atmosphere.Global greenhouse warming, on the timescale of 50 or 100 years is perceived as an immediate danger whereas the limits of the reserves (for coal) would only be felt after several centuries.

Fusion energy is the ultimate, $\mathrm{CO} 2$-free, resource for mankind, be it in the form of solar energy where and when sun shines, or from fusion processes confined in man-made machines. Fusion energy from the latter is relatively clean, producing, for the fusion reactions most easily controllable, only a limited amount of radioactive waste of moderate life-time.

Of the two confinement schemes known to date, Magnetic Confinement and Inertial Confinement, the first has been developed during the past fourty years to conditions that make thermonuclear burn and break-even a clear goal for the next generation of experimental Tokomac-type devices. Experiments towards Inertial Confinement Fusion (ICF) driven by laser beams were largely motivated by the need to understand the implosion mechanism in large-scale devices and to check the relevant computational tools, but provided the ground also for the present confidence in the feasibility of ICF. The solid-state-laser driven National Ignition Facility should advance this development to pellet ignition at small gain.

Heavy-ion drivers for ICF, the alternative to laser drivers, are recognized (ref.1) as the only scheme where reasonable extrapolations of present-day technology promise efficiency and reliability sufficient for an energy producing plant. These conjectures ought to be backed up by more data and oriented development work. It is in this optique that the development of a heavy-ion driven Ignition Facility has been undertaken.

\section{RECENT ACTIVITIES IN EUROPE}

\subsection{Driver development}

An exploratory investigation of the linac-plus-storage ring version of a heavy-ion driver was conducted in the early eighties and has led up to the HIBALL sytems studies concluded by 1985 (ref.2). Thereafter, studies of a variety of subjects oriented towards an eventual HIF facility were coordinated in a program "High Energy Density in Matter produced by Heavy Ion Beams" centered at the GSI laboratory at Darmstadt. A few of the subjects studied are listed below:

- The initial 'funneling' stages of a heavy ion linac, ion sources (ref.3) and

- RFQ accelerators for heavy ions (ref.4) and

- the merger of several stages with minimum loss of phase-space density into one main linac.

- A two-beam RFQ (Fig.1, ref.5).

- The IH structure, a new type of heavy-ion linac structure (ref.6).

- Focusing in the final beam transport by pulsed lenses (ref.7) or plasma lenses (ref.8, ).

- Charge-exchange cross-sections are being measured (ref.9) and beam losses in rings due to charge-exchange scattering computed(ref.10).

- Beam experiments at the CERN-PS, with a Laslett tuneshift of $\sim 1$ due to space-charge showed only small emittance blow-up(ref.11).

- In beam experiments at the GSI-ESR (ref.12), the beam remained stable at local densities exceeding the KeilSchnell criterium by a factor of 5 .

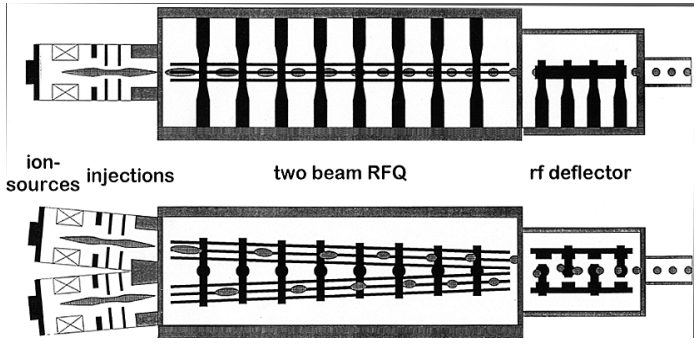

Figure 1: Two beam funneling experiment.

\subsection{Target physics}

A very substantial programme of computational target physics (beam-plasma interaction, opacities, radiation symmetrization in indirect drive targets, environmental problems, etc.) which cannot be presented within the limits of this paper, is under way in several institutions, e.g. DENIM (Madrid), ENEA (Frascati), MPI (Garching) as well as the Universities of Darmstadt and Frankfurt. 


\section{A TWO-STAGE PROGRAMME}

The current activities can be described in terms of a twostage programme:

- the GSI laboratory has launched an intensity upgrade project (ref.13) which will allow, by end 1998, filling of the GSI-SIS synchrotron with heavy ions up to its spacecharge limit, and

- a feasibility study is now under way of a heavy-ion driven Ignition Facility.

The GSI intensity upgrade project consists of three items to be implemented between 1995 and 1998, viz. the replacement of the first-stage Wideroe structure by a new front-end consisting of new ion sources, an RFQ and a modern IH structure, various improvements of the efficiency of the UNILAC linear accelerator, and the installation in the SIS synchrotron of an electron cooler. The number of ions per pulse produced to-day and the expected space-charge limit are shown in Fig. 2, whereas Fig. 3 shows the specific energy deposited and the plasma temperatures to be reached at the three stages.

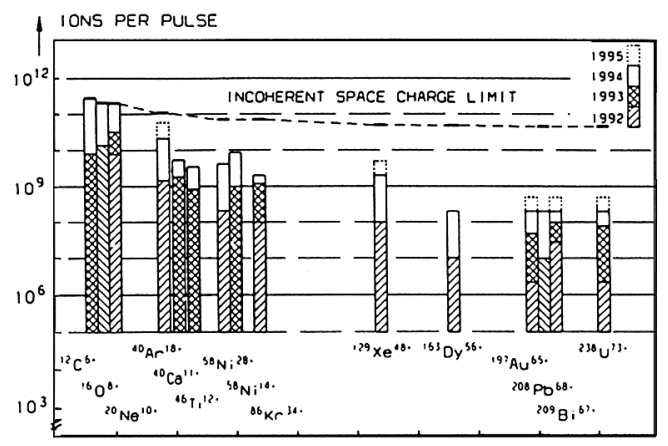

Fig. 2: Present \& expected ion beam intensities in SIS.

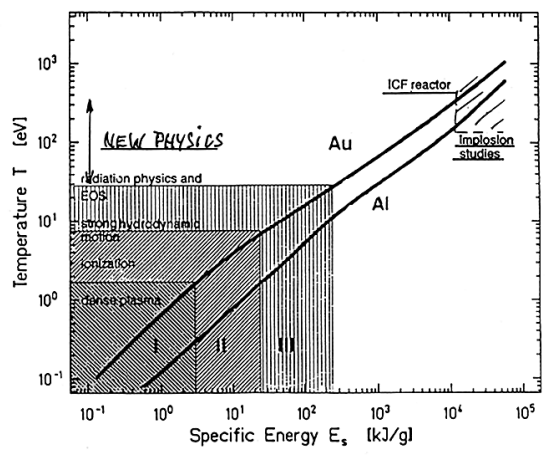

Figure 3: Plasma temperatures as a function of the specific energy deposition.

The Ignition Facility should, in a staged approach, bridge the gap (see Fig. 3) of two orders of magnitude in specific energy deposition, and one order of magnitude in plasma temperature, between the performance of the upgraded GSI machines and the parameters required for ignition of an ICF pellet. It shall serve the purposes of:
- the exploration of issues of accelerator design which cannot be tackled with existing machines,

- the exploration of a large programme of target physics,

- and finally, the demonstration of a heavy ion ICF driver and the demonstration of pellet ignition by heavy ions, in indirect drive mode, at moderate gain.

For the medium-term future, the Ignition Facility will provide the basis for the design of a Demonstration ICF Energy Plant.

\section{STUDY OF A HEAVY-ION DRIVEN IGNITION FACILITY}

This feasibility study (ref. 14) was launched by the management of four laboratories, i.e. DENIM (Madrid), ENEA (Frascati), GSI (Darmstadt), and KFK (Karlsruhe), and will very likely be supported by the European Union. Other laboratories with relevant experience are expected to join the collaboration.

Within the coming two to three years the feasibility and approximate cost shall be examined of a laboratory facility which ultimately can achieve ignition of DT pellets. The initial target parameters of the facility are given in the Table

\begin{tabular}{|l|l|}
\hline Ion energy & $6 \mathrm{GeV}$ (Bi ions) \\
\hline Ion range & $0.05 \mathrm{~g} / \mathrm{cm}^{2}$ \\
\hline Driver energy & $2 \mathrm{MJ}$ \\
\hline Beam pulse shape & $\begin{array}{l}\text { prepulse } 15-25 \mathrm{~ns}, 20 \mathrm{TW} \\
\text { main pulse } 6-7 \mathrm{~ns}, 300 \mathrm{TW}\end{array}$ \\
\hline Spot size & $2 \mathrm{~mm}, 2$ opposite converters \\
\hline Pointing tolerance & $.1-.2 \mathrm{~mm}$ \\
\hline Timing tolerance & $<.5 \mathrm{~ns}$ \\
\hline Target (indirect drive) & $\begin{array}{l}\text { pellet diameter } \leq 2 \mathrm{~mm} \\
\text { pellet mass } \leq 1.5 \mathrm{mg} \mathrm{DT}\end{array}$ \\
\hline Predicted gain & $10-30$ \\
\hline
\end{tabular}

and displayed in Fig. 4, but the final specifications and a reasonable staging, which is part of the charge to the study group, remain to be defined in interaction between driver and target experts. The facility must be considerably more modest than the power generating facilities considered in earlier systems studies: although the ion energy will be of the same order of magnitude, the total deposited beam energy is chosen such as to provide only for low target gain, and reaction chambers, beam spot dimensions, and (low) repetition rate will be adapted to laboratory studies. 


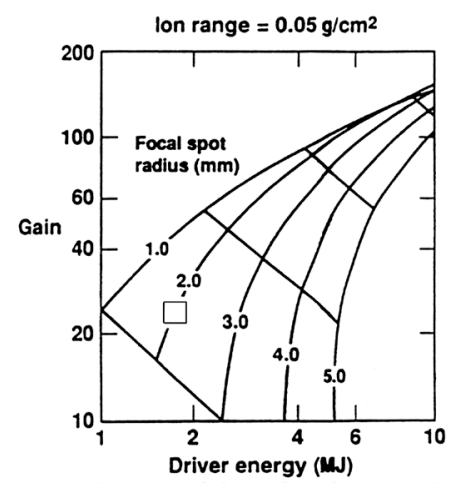

Figure 4: Gain curves for indirectly driven targets (Livermore group).

Recent advances and results do contribute to reducing the scale of the facility. It is now established that 'indirect drive' targets (Fig. 5) with only two converters at opposite ends can, with the help of internal screens, provide the necessary uniformity of pellet illumination (ref. 15, 16). The number of beams in final transport can then be chosen with respect to the Maschke transport limit, and the geometrical layout is simplified. Following accelerator experiments (ref. 12), it is expected that the Keil-Schnell criterium for longitudinal beam stability can be passed by about one order of magnitude in local beam density, for short dwelling times in any case. In other experiments, the beam was shown to survive for the time necessary for final bunching with small emittance blow-up (ref.11) even when integer transverse resonance lines were crossed due to space-charge. Routine operation (albeit at low intensity) of heavy ion linacs using the IH structure (ref. 17) indicates that the linac length may be considerably reduced.

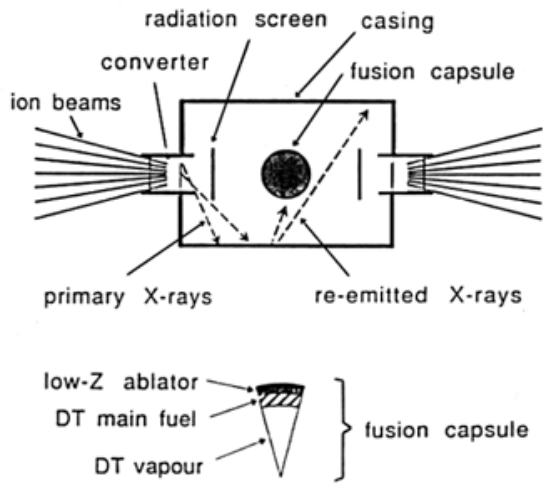

Figure 5: Indirect-drive target (ref. 15).

Conceptually, the facility will consist of one pulsed highcurrent linac $(\sim 100 \mathrm{~mA})$ fed by a funnel system (Ref. 18) of preaccelerators, in turn fed from 8 (possibly 16) ion sources, which injects into a small number of storage and buncher rings. The beam bunches of up to $10 \mathrm{~ns}$ duration are, through transport channels of different lengths, directed at the two converters of an indirect drive target, where they must impinge simultaneously.

Examination of the merits of various types of structures for the different stages of accelerators (RFQ, IH, SCDTL, etc.) has been launched along with the work on storage ring, bunching and final transport, so that a consistent set of accelerator parameters will become available in the near future.

\section{ACKNOWLEDGEMENTS}

This brief report summarizes many discussions with the coauthors of the ICF proposal (ref.14) and the persons responsible for the GSI project (ref.13), and in particular N. Angert, S. Atzeni, K. Blasche, R. Bock, D. Böhne, B. Franzke, I. Hofmann, H. Klein, and D. Möhl.

\section{REFERENCES}

1) Fusion Policy Advisory Cttee; Final report, Sept.1990; US DoE, Wash.DC,

2) HIBALL II, report KfK 3840 Kernforschungszentrum Karlsruhe, FRG

3) K. Volk et al., GSI 94-10 (1994) p. 34; GSI Darmstadt, FRG

4) H. Deitinghoff et al., GSI 94-10 (1994) p.33

5) A. Firjahn-Andersch et al. GSI 94-10, p.28

6) U. Ratzinger, Nuovo Cimento 106 A (1993) p. 1583

7) P. Spiller et al., Nuovo Cimento 106A (1993) p.1719

8) M. de Magistris, M. Stetter et al., Nuovo Cimento 106A (1993) p.1643,17256)

9) R. Schulze et al.,GSI 94-10 (1994) p.14; GSI Darmstadt, FRG

10) D.Budicin; I. Hofmann et al., Nuovo Cimento 106A (1993) p. 1621,1671

11) R. Cappi et al., Proc. EPAC 94 (1994) p. 279

12) I. Hofmann et al., Proc. EPAC 92 (1992) p. 783

13) D. Böhne et al. The GSI Intensity Upgrade Programme GSI 95-06 (1995)

14) S. Atzeni et al. Proposal for a Heavy Ion Ignition Facility GSI 95-03 (1995)

15) S. Atzeni, Nuovo Cimento 106A (1993) p.1489

16) J.D. Lindl, Nuovo Cimento 106A (1993) p.1467

17) H. Haseroth, this Conf.

18) A. Schempp, private comm. 